\title{
Entwicklungsbedeutung von Familienunternehmen für Transitionswirtschaften - Kommentar
}

\author{
Štefan Kajzer ${ }^{*}$
}

Im vorliegenden Heft befaßt sich die Autorin Dr. Mojca Duh mit den FamilienKMU's, die eine Schlüsselstellung in der entwickelten Wirtschaftswelt einnehmen. Schon allein dadurch stellen sie einen bedeutenden Entwicklungsfaktor dar. Wie die Autorin aufgrund der statistischen Wirtschaftsdaten und ihrer Untersuchungen richtig feststellt, bekommen diese KMU's besonders in den Transitionsländern in ihren Umstrukturierungsprozessen eine entscheidende Rolle. Da die Familienunternehmen in diesen Ländern wegen ihrer Wirtschaftsgeschichte natürlich »noch in den Kinderschuhen stecken«, handelt es sich überall dort um ein echtes wirtschaftliches und natürlich auch gesellschaftliches Problem. Um eine wirtschaftlich annehmbare Lösung zu finden, befaßt sich die Autorin zuerst mit der Rolle und Bedeutung von Familienunternehmen für Wirtschaft und Gesellschaft und dann mit einigen Erkenntnissen über Familien-KMU's in Slowenien, die vorwiegend aus eigenen Untersuchungen dieses Phänomens hervorgehen. Daraus versucht sie die wichtigsten Schlußfolgerungen zu ziehen.

Im ersten Teil ihres Beitrags setzt sich die Autorin zuerst mit verschiedenen wirtschaftlichen und gesellschaftlichen Dimensionen der Familienunternehmen auseinander. Dabei basieren die meisten Feststellungen auf den Erkenntnissen vieler Untersuchungen in den Ländern mit einer entwickelten Marktwirtschaft, d. h. aus den wirtschaftlichen und gesellschaftlichen Verhältnissen, die die Transitionsländer anstreben. Wie sie richtig feststellt, wird man gerade in diesen Ländern der Entwicklungsrolle der Familienunternehmen noch nicht gerecht und ist daher nicht bemüht, unterstützende Entwicklungsbedingungen zu schaffen. Sie setzt sich berechtigt dafür ein, daß »dabei die Entwicklungsfragen der Familien-KMU's, ihre Rolle innerhalb der Gesamtentwicklung des einzelnen Landes und die Besonderheiten des Entwicklungsmanagements dieser Unternehmen im Zentrum stehen müssen«. Es fehlt vielleicht die Beurteilung der Bedeutung der Familien-KMU's in den Globalisierungsprozessen. Wie die diesbezüglichen Untersuchungen andeuten, verlieren diese Unternehmen durch diese Prozesse nicht an wirtschaftlicher (und gesellschaftlicher) Bedeutung.

\footnotetext{
* Universität Maribor, Fakultät für Wirtschaftswissenschaften;
} 
Den zweiten Teil des Beitrags widmet die Autorin den Erkenntnissen über Familien-KMU's in Slowenien. Dabei muß man betonen, daß die Autorin in den diesbezüglichen theoretischen und empirischen Untersuchungen aktiv mitgewirkt hat und daß ihre Dissertation »Entwicklungsbesonderheiten des Familienunternehmens « das wichtigste Werk zu diesem Thema in Slowenien darstellt. Im Beitrag faßt sie nur die wichtigsten Ergebnisse ihrer Untersuchungen zusammen, sie genügen aber doch, um sich ein klares Bild über die diesbezügliche Situation in Slowenien zu schaffen. Es hat sich gezeigt, daß in den neunziger Jahren die Anzahl der KMU sprunghaft angestiegen, ihr Anteil aber im Vergleich zu den effektivsten Weltwirtschaften immer noch zu klein ist. Detailliert werden im Beitrag die Ergebnisse der Untersuchung der FamilienKMU's in einer der Regionen Sloweniens gezeigt und bewertet. Da es in Slowenien zur Zeit noch keine vollständige Datenbasis über die Familienunternehmen gibt, lassen sich die Regionenverhältnisse nur mit einigem Risiko auf das ganze Land verallgemeinern.

Im Schlußteil ihres Beitrags setzt sich die Autorin dafür ein, daß man in allen Transitionsländern »die Besonderheiten der Entwicklung der Familien-KMU's zuerst (er)kennen, sich ihrer bewußt werden und sie auf dieser Grundlage entsprechend unterstützen muß«.

Die Autorin präsentiert in ihrem Beitrag die Ergebnisse theoretischer und empirischer Untersuchungen zum Problem der Entwicklung von Familienunternehmen in den Transitionswirtschaften. Daraus leitet sie einige durchaus empfehlenswerte Schlußfolgerungen $a b$, die für die Länder mit erst entstehenden Familien-KMU's von großer Bedeutung und im Prinzip in allen diesen Wirtschaften anwendbar sind. Sie beruft sich im Beitrag korrekt auf die für das erörterte Problem relevante Fachliteratur, auf eigene Arbeiten darüber und auf andere Datenquellen, die in der Untersuchung benutzt wurden. 